\title{
Caminhos e descaminhos da Ortodontia no Brasil
}

\author{
Anna Paula MORGENSTERN*, Marco Antonio Lopes FERES**, Eros PETRELLI***
}

\begin{abstract}
Resumo
Devido à constatação do aumento do número de ortodontistas e de Cursos de Ortodontia, delineou-se este estudo cujo objetivo foi sondar o futuro da especialidade. Avaliaram-se formulários respondidos por 413 alunos e 130 professores de 42 Cursos de Especialização dos 73 relacionados no Catálogo da Associação Brasileira de Ortodontia e Ortopedia Facial (ABOR) 1999/2000. Obteve-se uma taxa de resposta de 57,53\%. A amostra foi dividida em: alunos e professores e nas regiões: Sul + Sudeste e Centro-Oeste + Nordeste. A mediana de idade do pós-graduando foi 30,8 e a do professor 42,8 anos. O índice preconizado pela Organização Mundial de Saúde é de 1 CD para 1.500 habitantes. O Brasil com uma população de 175 milhões e 175.637 dentistas, apresenta 1 CD para 996 habitantes. O Reino Unido apresenta 1/2.000, a França 1/1.519, e a Itália 1/1.333 (1998). Com população 40\% e renda 82\% menor do que os EUA, o Brasil possui quase o triplo de cursos de Ortodontia (n=124, 2001), graduando 344 novos ortodontistas/ ano. Considerando-se os 175.637 dentistas, o número de especialistas ainda é pequeno no Brasil. Para a maioria dos entrevistados, o mercado de trabalho foi considerado pessimista/saturado $(58,7 \%)$ devido aos cursos não oficiais $(85,1 \%)$ e à concorrência por clínicos-gerais $(63,4 \%)$. Medidas proibitivtas de cursos não regulamentados foram desejáveis pela maior parte $(88,3 \%)$. Os honorários foram considerados insuficientes para $44,8 \%$ e satisfatórios para 47,3\%. O incremento da renda é feito com a clínica-geral $(58,2 \%)$ pelos alunos e com as atividades docentes $(73,3 \%)$ pelos professores. A maioria não estimularia seu filho a estudar Odontologia hoje $(66,9 \%)$ e a principal fonte de indicação provém dos pacientes $(89,3 \%)$. Outro notável achado foi que as documentações intermediárias e finais não estão sendo realizadas por $82,8 \%$ dos ortodontistas.
\end{abstract}

Palavras-chave: Ortodontistas brasileiros. Mercado de trabalho. Gerenciamento de consultório. Marketing.

\section{INTRODUÇÃO}

Nos últimos anos têm-se observado um aumento dos especialistas e de cursos de Ortodontia, o que gerou uma expectativa junto à comunidade ortodôntica: para onde a Ortodontia seguirá o seu caminho e de que maneira sinalizará seu futuro? Delinearam-se assim, os objetivos da pesquisa: sondar o futuro da especialidade, indicar tendências comportamentais, avaliar as perspectivas de mercado e demonstrar métodos de gerenciamento e marketing.

* Pós-graduada em Ortodontia e Ortopedia Facial pela Universidade Federal do Paraná.

** Professor Adjunto IV da Disciplina de Ortodontia da Universidade Federal do Paraná

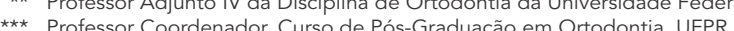




\section{REVISÃO DE LITERATURA}

Panorama nacional

De acordo com a tabela 1, o Brasil gradua 344,6 ortodontistas por ano.

\section{$\mathrm{CD} /$ habitantes - Brasil}

A tabela 2 descreve a relação de $\mathrm{CD}$ / habitantes do Brasil.

Números da Odontologia X Ortodontia - Brasil

Do número total de dentistas do Brasil (175.637), 2,2\% correspondem a ortodontistas. A participação de ortodontistas no Brasil é quase 4 vezes menor (9\%) no Paraná do que em São Paulo (40\%), isto é, São Paulo tem 4,5 vezes mais ortodontistas que o Paraná, conforme a tabela 3 e o gráfico 1 .

Petrelli ${ }^{27}$ fez um alerta aos profissionais: somente cursos realizados em Universidades, Faculdades e Entidades de Classe têm reconhecimento do Conselho Federal de Odontologia e validade de seus certificados.

Petrellii ${ }^{28}$ declarou que cursos irregulares são acobertados sob a sombra de entidades denominados núcleos, institutos ou centro de estudos. São cursos denominados espúrios, sem o amparo legal, cuja principal finalidade é o lucro financeiro particular.

Koubik e Feres ${ }^{20}$ relacionaram caber às Associações de Classe: 1) promover discussão ampla da responsabilidade profissional; 2) combater cursos ineficientes de interesses mercantilistas; e 3) alertar a opinião pública sobre quais os profissionais realmente capacitados a exercer Ortodontia.

Feres $^{10}$ simplificou a conduta profissional: 1) saber dizer não, 2) "jogar fora" metade de seu consultório, 3) planejar a mecânica como um cronograma, 4) ter cuidado com o período da Dentição Mista, 5) solicitar radiografias freqüentes, 6) concentrar-se no foco, e 7) informar ao paciente, antes, todos os aspectos pertinentes ao tratamento.

Haag ${ }^{16}$ conclui que: 1) nenhum programa de Graduação dá condições do exercício pleno da especialidade e 2) a suspensão de abertura de

\begin{tabular}{|c|c|c|c|c|}
\hline \multicolumn{5}{|c|}{ Tabela 1 - Evolução do ${ }^{0}$ de ortodontistas - Brasil } \\
(Fonte: CFO).
\end{tabular}

\begin{tabular}{|c|c|c|}
\hline \multicolumn{3}{|c|}{ Tabela 2 - CD/ habitantes - Brasil. } \\
\hline ÍNDICE & CD/ HABITANTES & FONTE \\
\hline OMS & $1 / 1500$ & CRO/PR 7,1999 \\
\hline BRASIL & $1 / 996$ & CFO e IBGE ${ }^{6}, 1996$ \\
\hline $\mathrm{SP}$ & $1 / 635$ & LIMA $^{24}, 2001$ \\
\hline PR & $1 / 700$ & CRO/PR7, 1999 \\
\hline CURITIBA & $1 / 400$ & CRO/PR7, 1999 \\
\hline
\end{tabular}

\begin{tabular}{|c|c|c|c|c|}
\hline & Brasil & SP & PR & Fonte \\
\hline $\mathrm{N}^{0}$ dentistas & 175.637 & 60.224 & 10.515 & (LIMA $\left.^{25}, 2001\right)$ \\
\hline $\begin{array}{l}\text { Cursos Odon- } \\
\text { tologia }\end{array}$ & mais 100 & mais 40 & & $\begin{array}{l}\left(\text { LIMA }^{24,25},\right. \\
2001)\end{array}$ \\
\hline $\begin{array}{l}\mathrm{N}^{0} \text { ortodon- } \\
\text { tistas }\end{array}$ & 3.831 & 1.362 & 305 & (CFO $\left.^{6}, 2001\right)$ \\
\hline $\begin{array}{l}\text { Cursos } \\
\text { Ortodontia }\end{array}$ & 124 & 47 & 11 & (CFO $\left.^{6}, 2001\right)$ \\
\hline $\begin{array}{l}\text { Orto/Odon- } \\
\text { tológos }\end{array}$ & $2,2 \%$ & $2,3 \%$ & $2.9 \%$ & \\
\hline
\end{tabular}

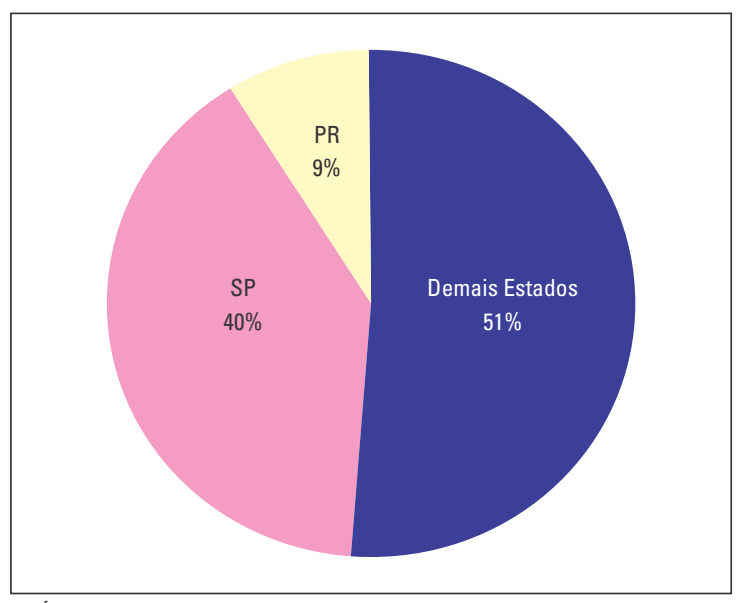

GRÁFICO 1 - Participação dos ortodontistas no Brasil. 
cursos de Ortodontia ocasionou a promoção de typodonts, apesar do único requisito para a aprovação nos cursos registrados no $\mathrm{CFO}$ ser o registro no CRO. Não existe lei que impeça a prática da Ortodontia por profissional não especialista, o que pode gerar complicações no decorrer de um tratamento.

Segundo Cauduro ${ }^{4}$, as áreas que ainda possibilitam novos entrantes são a Ortodontia e/ou Ortopedia, a Laserterapia e a Implantodontia: são substitutas (menos a Ortodontia) e possuem fornecedores com materiais altamente depreciados.

De acordo com Petrelli ${ }^{29}$, o Provão tem como objetivo contribuir para o diagnóstico do ensino da Odontologia no Brasil, pela avaliação do desempenho de seus graduandos.

Conforme o $\mathrm{CFO}^{5}$, a Odontologia brasileira possui 14 especialidades e 423 cursos de Especialização em andamento para atender 29.144 especialistas, que correspondem a $17,74 \%$ dos cirurgiões-dentistas inscritos nos CROs.

Segundo Isidoro ${ }^{17}$, enquanto a obrigação do Ministério da Educação é zelar pela boa qualidade do ensino, o exercício da profissão deveria ser regulamentado pelo $\mathrm{CFO}$.

Para Faltin Junior ${ }^{9}$, a criação da Ortopedia é um retalhamento dos métodos terapêuticos da nossa especialidade, e é exclusivamente político.

\begin{tabular}{|c|c|c|c|}
\hline \multicolumn{3}{|c|}{ Tabela 4 - CD/ habitantes - Brasil/países europeus ${ }^{37}$} \\
\hline País & $\mathbf{N}^{\circ} \mathbf{C D}$ & População & $\mathbf{C D} / \mathbf{H a b}$. \\
\hline $\begin{array}{c}\text { Brasil } \\
\text { Reino Unido } \\
\text { (1998) }\end{array}$ & 175.637 & 175 milhões & $1 / \mathbf{9 9 6}$ \\
\hline $\begin{array}{c}\text { França (1998) } \\
\text { Itália (1998) }\end{array}$ & 30.000 & 60 milhões & $1 / \mathbf{2 . 0 0 0}$ \\
\hline
\end{tabular}

\section{Panorama internacional}

$\mathrm{CD} /$ Habitantes - Brasil/Países europeus

A tabela 4 compara a relação $\mathrm{CD}$ / habitantes no Brasil e nos países europeus.

De acordo com a tabela 5, o Brasil com uma população $40 \%$ menor (161,8 milhões) que a dos EUA ( 273,8 milhões) e renda $82 \%$ menor, possui mais que o dobro de cursos. Até 2001, eram 124 de Ortodontia ${ }^{8}$ e 160 de Odontologia ${ }^{24,25}$, enquanto os EUA possuíam até 1995, 53 de Ortodontia ${ }^{36}$ e 46 de Odontologia ${ }^{21}$.

Johnson, Gottlieb e Domer ${ }^{18}$ observaram que os ortodontistas americanos com maior renda são os que possuem maior números de casos ortodônticos, empregam mais funcionários, delegam mais funções e utilizam metodologia gerencial.

Gottlieb, Nelson e Vogels $s^{12,14,15}$ declararam que o crescimento profissional nos EUA foi atribuído à adoção de técnicas administrativas, sendo considerado o maior obstáculo o aumento do número de generalistas exercendo a Ortodontia.

Para Sullivan ${ }^{35}$, deve-se associar atividade com produtividade e rentabilidade, diminuindo-se o número de emergências, faltas, consultas antes do tempo e de pacientes já finalizados.

Fox et al. ${ }^{11}$ avaliaram 375 pares de modelos iniciais e 250 finais de 41 clínicos-gerais. Os ortodontistas qualificados obtiveram melhores resultados com aparelhos fixos do que os sem qualificação.

Para Richmond et al. ${ }^{32}$ as principais dificuldades enfrentadas pela Ortodontia na Inglaterra incluem a relativa alta demanda pelo tratamento ortodôntico, número insuficiente de ortodontistas especialistas, distribuição geográfica irregular dos profissionais e problemas com a remuneração.

\begin{tabular}{|c|c|c|c|c|c|}
\hline \multicolumn{6}{|c|}{ Tabela 5 - Cursos X População - Brasil e Estados Unidos } \\
\hline País & $\begin{array}{l}\text { Pop. em } \\
\text { milhões }\end{array}$ & Cursos Odontologia & Cursos Ortodontia & População Urbana & Renda/ capita (dólares) \\
\hline EUA & 273,8 & 46 & $\begin{array}{c}53 \\
\left.\text { (WALDMAN }^{36}, 1998\right)\end{array}$ & $76 \%$ & 28.020 \\
\hline BRASIL & 161,8 & $\begin{array}{c}160 \\
\left(\text { LIMA }^{24,25}, 2001\right)\end{array}$ & $\begin{array}{c}124 \\
\left.\text { (CAPES }{ }^{8}, 2001\right)\end{array}$ & $78 \%$ & 5.029 \\
\hline
\end{tabular}


Stratford e Burden ${ }^{34}$ avaliaram 167 modelos ortodônticos iniciais e finais de 18 clínicos-gerais e constataram a necessidade de retratamento em $41,5 \%$. Os clínicos-gerais deveriam tratar pacientes que requeressem intervenção ortodôntica menos complexa.

Bergström et al. ${ }^{3}$ estudaram 313 pacientes ortodônticos tratados por clínicos-gerais e especialistas na Suécia. Os especialistas forneceram três vezes mais tratamentos difíceis e os resultados foram mais favoráveis.

Waldman ${ }^{36}$ concluiu que durante os anos 90, a diminuição da taxa de ortodontistas por jovens nos EUA, refletiu: 1) um aumento da população entre 5 a 19 anos, e 2) uma diminuição no número de ortodontistas.

\section{MATERIAIS E MÉTODOS}

A metodologia foi a mesma aplicada aos trabalhos anteriormente realizados pelo Curso de Pósgraduação em Ortodontia e Ortopedia Facial da UFPR $^{30,31,33}$, através da aplicação de um questionário com 20 perguntas em alunos e professores dos 73 Cursos de Especialização em Ortodontia relacionados no Catálogo da Associação Brasileira de Ortodontia (ABOR) 1999/20002.

Avaliou-se o mercado atual, o gerenciamento do consultório, a renda, abordagens de tratamento e marketing.

\section{RESULTADOS}

Com uma taxa de resposta de $57,53 \%$, a tabela 6 descreve os números obtidos entre as regiões:

Para efeitos comparativos, dividiu-se a amostra

\begin{tabular}{|c|c|c|c|}
\hline \multicolumn{3}{|c|}{ Tabela $\mathbf{6}-$ No. de escolas, alunos e professores por região } \\
\hline Regiões & $\mathbf{N}^{\mathbf{0}}$ de Escolas & $\mathbf{N}^{\mathbf{0}}$ de Alunos & $\begin{array}{c}\mathbf{N}^{\mathbf{0}} \text { de Profes- } \\
\text { sores }\end{array}$ \\
\hline SU & 9 & 82 & 32 \\
\hline SE & 26 & 271 & 74 \\
\hline CO & 3 & 30 & 8 \\
\hline NE & 4 & 30 & 16 \\
\hline Total & $\mathbf{4 2}$ & $\mathbf{4 1 3}$ & $\mathbf{1 3 0}$ \\
\hline
\end{tabular}

em 2 regiões: 1) Sul + Sudeste; e 2) Nordeste + Centro-Oeste e separou-se em 2 grupos: 1) Alunos e 2) Professores. A $1^{\text {a }}$ região englobou os Estados: Minas Gerais, Rio de Janeiro, São Paulo, Paraná, Rio Grande do Sul e Santa Catarina; e a $2^{\mathrm{a}}$ região: Ceará, Paraíba, Pernambuco, Goiás e Mato Grosso.

Os resultados estão dispostos nos quadros 1 a 3 , nas tabelas 7 e 8 e nos gráficos 2 a 9 . Os quadros mostram as porcentagens calculadas sobre as respostas desconsiderando as respostas em branco.

\section{Análise dos alunos}

$\mathrm{Na}$ comparação dos alunos foram constatadas as seguintes significâncias estatísticas para:

\section{Grupo 1 (Sul+Sudeste)}

- A maioria $(86,6 \%)$ dos profissionais não está documentando seus casos durante fases intermediária e final $(\mathrm{p}=0,0180)$;

\section{Grupo 2 (Centro-Oeste+Nordeste)}

- Buscam para o incremento de sua renda, a clínica-geral ou outras especialidades $(58,2 \%)$ $(\mathrm{p}=0,0493)$;

- Os honorários atuais cobrados são satisfatórios $(58,6 \%)(\mathrm{p}=0,0461)$;

- São mais velhos $(30,7 \pm 5,3 \times 31,4 \pm 4,7)$ $(\mathrm{p}=0,027)$;

- Estão formados a mais tempo $(8,0 \pm 5,1 \mathrm{x}$ $8,8 \pm 5,0)(\mathrm{p}=0,009)$;

- E são especialistas em Ortodontia a menos tempo $(3,8 \pm 3,2 \times 2,5 \pm 2,1)(\mathrm{p}<0,0001)$.

\section{Análise dos professores}

$\mathrm{Na}$ comparação dos professores foi constatada diferença significativa apenas para o Grupo 2, onde:

- Vêem o mercado atual como pessimista e/ou saturado $(62,5 \%)(p=0,0283)$.

\section{Alunos $\mathrm{x}$ professores}

Mercado de Trabalho (Quadro 1), Tratamento e Cliente (Quadro 2), Marketing (Tab. 7), Documentação (Tab. 8), Idade e Tempo de Formado e 


\begin{tabular}{|c|c|c|c|c|c|c|}
\hline \multicolumn{7}{|c|}{ Quadro 1 - Dados gerais dos profissionais de Ortodontia em relação ao mercado de trabalho. } \\
\hline \multirow[t]{2}{*}{ DADOS } & \multicolumn{2}{|c|}{$\begin{array}{l}\text { ALUNOS } \\
(n=413)\end{array}$} & \multicolumn{2}{|c|}{$\begin{array}{l}\text { PROFESSORES } \\
\quad(\mathrm{n}=130)\end{array}$} & \multicolumn{2}{|c|}{$\begin{array}{l}\text { TOTAL } \\
\text { (n = 543) }\end{array}$} \\
\hline & N. ${ }^{0}$ & $\%$ & N. ${ }^{\circ}$ & $\%$ & N. ${ }^{0}$ & $\%$ \\
\hline Como você vê o mercado atual & 400 & 96,9 & 130 & 100,0 & 530 & 97,6 \\
\hline - Otimista / Absorve mais recursos humanos & 31 & 7,7 & 08 & 6,1 & 39 & 7,3 \\
\hline • Estável & 134 & 33,5 & 46 & 35,4 & 180 & 34,0 \\
\hline - Pessimista / Saturado & 235 & 58,8 & 76 & 58,5 & 311 & 58,7 \\
\hline Causas de saturação do mercado & 1372 & 3,3/entr & 420 & 3,2/entr & 1792 & 3,3/entr \\
\hline - Grande número de cursos não oficiais & 358 & 86,7 & 104 & 80,0 & 462 & 85,1 \\
\hline - Concorrência por ortodontistas especialistas & 33 & 8,0 & 21 & 16,2 & 54 & 9,9 \\
\hline - Concorrência por clínicos-gerais & 320 & 77,5 & 99 & 76,2 & 419 & 77,2 \\
\hline - Situação econômica do país desfavorável & 258 & 62,5 & 86 & 66,2 & 344 & 63,4 \\
\hline - Falta de união da Classe odontológica e ortodôntica & 236 & 57,1 & 65 & 50,0 & 301 & 55,4 \\
\hline • Excessivo número de Cursos de Pós-Graduação & 167 & 40,4 & 45 & 34,6 & 212 & 39,0 \\
\hline Controle de cursos não oficiais por Entidades e Órgãos & 409 & 99,0 & 129 & 99,2 & 538 & 99,1 \\
\hline$\cdot \operatorname{Sim}$ & 373 & 91,2 & 100 & 77,5 & 473 & 87,9 \\
\hline • Não & 36 & 8,8 & 29 & 22,5 & 65 & 12,1 \\
\hline Medidas proibitivas de cursos não regulamentados & 409 & 99,0 & 128 & 98,5 & 537 & 98,9 \\
\hline • $\operatorname{Sim}$ & 368 & 90,0 & 106 & 82,8 & 474 & 88,3 \\
\hline • Não & 41 & 10,0 & 22 & 17,2 & 63 & 11,7 \\
\hline Soluções / alternativas para o incremento da renda & 371 & 89,8 & 116 & 89,2 & 487 & 89,7 \\
\hline - Atividades docentes & 44 & 11,9 & 85 & 73,3 & 129 & 26,5 \\
\hline - Gerenciamento de consultório (s) & 112 & 30,2 & 41 & 35,3 & 153 & 31,4 \\
\hline - Investimentos em outras áreas do mercado & 79 & 21,3 & 50 & 43,1 & 129 & 26,5 \\
\hline - Exercício de clínica-geral ou outras especialidades & 206 & 55,5 & 07 & 6,0 & 213 & 43,7 \\
\hline • Total & 441 & 1,2/entr & 183 & 1,6/entr & 624 & $1,3 /$ entr \\
\hline É correto & 401 & 97,1 & 127 & 97,7 & 528 & 97,2 \\
\hline - Estipular valores de aparelho & 178 & 44,4 & 34 & 26,8 & 212 & 40,2 \\
\hline - Estipular valores para o tratamento como um todo & 225 & 56,1 & 100 & 78,7 & 325 & 61,6 \\
\hline - Estipular mensalidades sem entrada & 31 & 7,7 & 07 & 5,5 & 38 & 7,2 \\
\hline • Total & 434 & 1,1/entr & 141 & 1,1/entr & 575 & $1,1 /$ entr \\
\hline Os honorários atuais cobrados & 405 & 98,1 & 126 & 96,9 & 531 & 97,8 \\
\hline -Insuficientes & 188 & 46,4 & 50 & 39,7 & 238 & 44,8 \\
\hline - Satisfatórios & 186 & 45,9 & 65 & 51,6 & 251 & 47,3 \\
\hline - Bons & 31 & 7,7 & 11 & 8,7 & 42 & 7,9 \\
\hline Estimularia seu filho a estudar Odontologia hoje? & 402 & 97,3 & 129 & 99,2 & 531 & 97,8 \\
\hline - $\operatorname{Sim}$ & 121 & 30,1 & 55 & 42,6 & 176 & 33,1 \\
\hline • Não & 281 & 69,9 & 74 & 57,4 & 355 & 66,9 \\
\hline
\end{tabular}

NOTA: GRUPO 1 = Alunos; GRUPO 2 = Professores. 
Quadro 2 - Dados gerais dos profissionais de Ortodontia em relação ao tratamento e cliente.

\section{DADOS}

Tempo médio de tratamento com aparelhagem fixa (meses)

$\cdot 18$

- Mais de 36

Abordagem em relação ao paciente com dentição mista

- Trata em uma fase

- Trata em duas fases

- Aguarda a dentição permanente

- Outra

- Total

Principal fonte de indicação de novos pacientes

- Próprios pacientes

- Colegas

- Ex-alunos

- Amigos

- Indicadores ou anúncios

- Encontros e/ou reuniões sociais

- Total

ALUNOS

( $n=413$ )

N. ${ }^{\circ} \quad \%$

$09 \quad 2,6$

$153 \quad 44,0$

$161 \quad 46,3$

$22 \quad 6,3$

398

398

$31-7,8$

$343 \quad 86,2$

$12 \quad 3,0$

26

412

6,5

654

1,6/entr

PROFESSORES

N. ${ }^{0}$

= 130)

TOTAL

( $n=543$ )

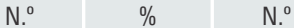

$\%$

\begin{tabular}{l|l|l|l|}
\hline 129 & 99,2 & 477 & 87,8 \\
\hline
\end{tabular}

\begin{tabular}{|l|l|l|l}
\hline 08 & 6,2 & 17 & 3,6 \\
\hline
\end{tabular}

\begin{tabular}{|l|l|l|l|}
\hline 46 & 35,6 & 199 & 41,7 \\
\hline
\end{tabular}

\begin{tabular}{|l|l|l|l|l|}
\hline 66 & 51,2 & 227 & 47,6 \\
\hline
\end{tabular}

\begin{tabular}{|l|l|l|l|l|}
\hline 6,3 & 09 & 7,0 & 31 & 6,5 \\
\hline
\end{tabular}

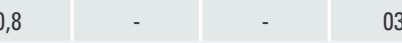

0,6

\begin{tabular}{|c|c|c|c|c|c|}
\hline 403 & 97,6 & 129 & 99,2 & 532 & 98,0 \\
\hline 359 & 89,1 & 116 & 89,9 & 475 & 89,3 \\
\hline 173 & 42,9 & 65 & 50,4 & 238 & 44,7 \\
\hline 06 & 1,5 & 20 & 15,5 & 26 & 4,9 \\
\hline 85 & 21,1 & 25 & 19,4 & 110 & 20,7 \\
\hline 21 & 5,2 & 13 & 10,1 & 34 & 6,4 \\
\hline 10 & 2,5 & 04 & 3,1 & 14 & 2,6 \\
\hline
\end{tabular}

Casuística no tratamento de adultos (percentual)

\begin{tabular}{l|l|l}
243 & $1,9 /$ entr & 897
\end{tabular}

1,7/entr

$\bullet 10$

- 20

$\cdot 30$

- 40

- 50 ou mais

\begin{tabular}{|c|c|c|c|c|c|}
\hline 361 & 87,4 & 117 & 90,0 & 478 & 88,0 \\
\hline 53 & 14,7 & 13 & 11,1 & 66 & 13,8 \\
\hline 57 & 15,8 & 24 & 20,5 & 81 & 17,0 \\
\hline 104 & 28,8 & 38 & 32,5 & 142 & 29,7 \\
\hline 83 & 23,0 & 28 & 23,9 & 111 & 23,2 \\
\hline 64 & 17,7 & 14 & 12,0 & 78 & 16,3 \\
\hline
\end{tabular}

Clínica mais eficiente em relação à duração do tratamento

\begin{tabular}{|l|l|l|l|l|r|r|}
\hline 368 & 89,1 & 127 & 97,7 & 495 & 91,2 \\
\hline
\end{tabular}

- Utiliza aparelho pré-ajustado

- Trabalha com pessoal auxiliar

- Trabalha com várias cadeiras ao mesmo tempo

- Utiliza notificação por escrito

- Toma atitude forte em relação às faltas

- Delega tarefas

\begin{tabular}{c|c|c|c|c|c|}
\hline 232 & 63,0 & 66 & 52,0 & 298 & 60,2 \\
\hline 184 & 50,0 & 75 & 59,1 & 259 & 52,3 \\
\hline 66 & 17,9 & 43 & 33,9 & 109 & 22,0 \\
\hline 188 & 51,1 & 64 & 50,4 & 252 & 50,9 \\
\hline 106 & 28,8 & 42 & 33,1 & 148 & 29,9 \\
\hline 70 & 19,0 & 21 & 16,5 & 91 & 18,4 \\
\hline 846 & & & & & \\
\hline
\end{tabular}

- Total

2,3/entr 
Tabela 7- Instrumentos de Marketing utilizados pelos profissionais de Ortodontia.

\begin{tabular}{|c|c|c|c|c|c|c|}
\hline \multirow[t]{2}{*}{ TIPOS DE INSTRUMENTOS } & \multicolumn{2}{|c|}{ ALUNOS } & \multicolumn{2}{|c|}{ PROFESSORES } & \multicolumn{2}{|c|}{ TOTAL } \\
\hline & N. ${ }^{0}$ & $\%$ & N. ${ }^{\circ}$ & $\%$ & N. ${ }^{\circ}$ & $\%$ \\
\hline Mala direta & 137 & 36,0 & 22 & 17,6 & 159 & 31,4 \\
\hline Correspondências em datas festivas & 173 & 45,4 & 45 & 36,0 & 218 & 43,1 \\
\hline Logomarca & 95 & 24,9 & 25 & 20,0 & 120 & 23,7 \\
\hline Indicadores profissionais & 166 & 43,6 & 65 & 52,0 & 231 & 45,7 \\
\hline Outros & 75 & 19,7 & 30 & 24,0 & 105 & 20,8 \\
\hline - Boca a boca & 33 & 44,0 & 17 & 56,7 & 50 & 47,6 \\
\hline - Qualidade do trabalho & 13 & 17,3 & 09 & 30,0 & 22 & 21,0 \\
\hline - Brindes & 03 & 4,0 & 01 & 3,3 & 04 & 3,8 \\
\hline -Palestras & 10 & 13,3 & 03 & 10,0 & 13 & 12,4 \\
\hline • Meios de comunicação & 21 & 28,0 & 05 & 16,7 & 26 & 24,8 \\
\hline - Colegas & 09 & 12,0 & 07 & 23,3 & 16 & 15,2 \\
\hline - Terceiros & 03 & 4,0 & 01 & 3,3 & 04 & 3,8 \\
\hline - Total & 92 & $1,2 /$ entr & 43 & 1,4 / entr & 135 & $1,3 /$ entr \\
\hline TOTAL GERAL & $646 / 381$ & $1,7 /$ entr & $187 / 125$ & $1,5 /$ entr & $833 / 506$ & $1,6 /$ entr \\
\hline
\end{tabular}

NOTA: Percentual calculado em razão do número de casos relatados.

Tabela 8 - Falta de documentação dos casos durante fases intermediárias e final por parte dos profissionais de Ortodontia.

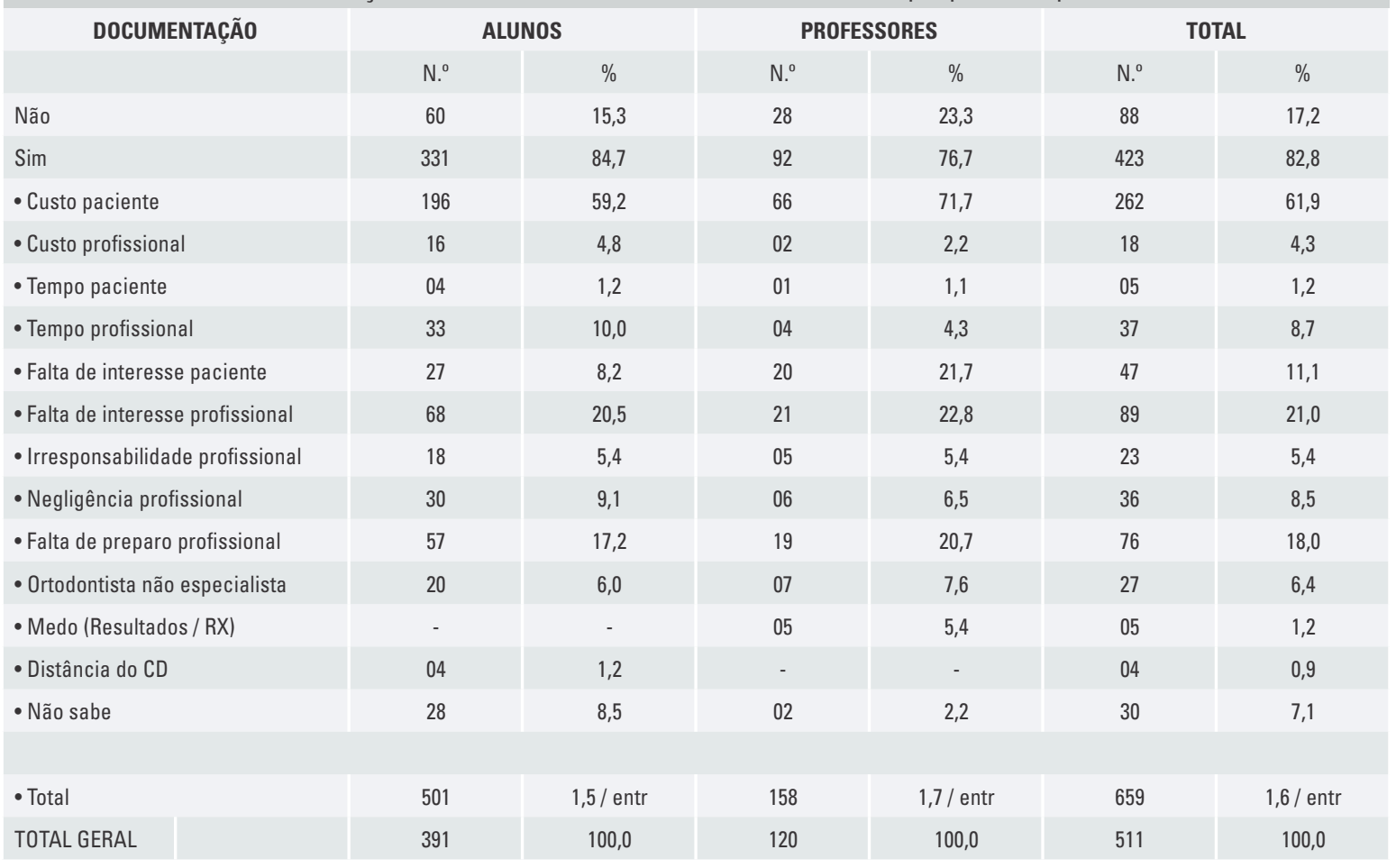

NOTA: Percentual calculado em razão do número de casos relatados. 


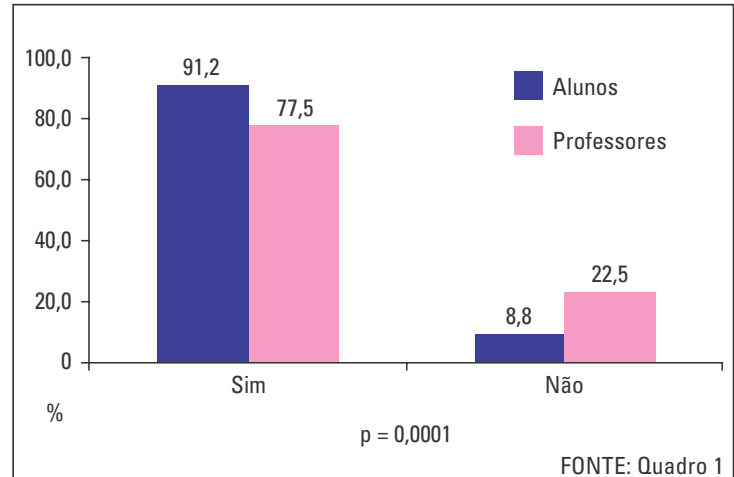

GRÁFICO 2 - Controle de cursos não oficiais por entidades e órgãos em relação aos alunos e professores.

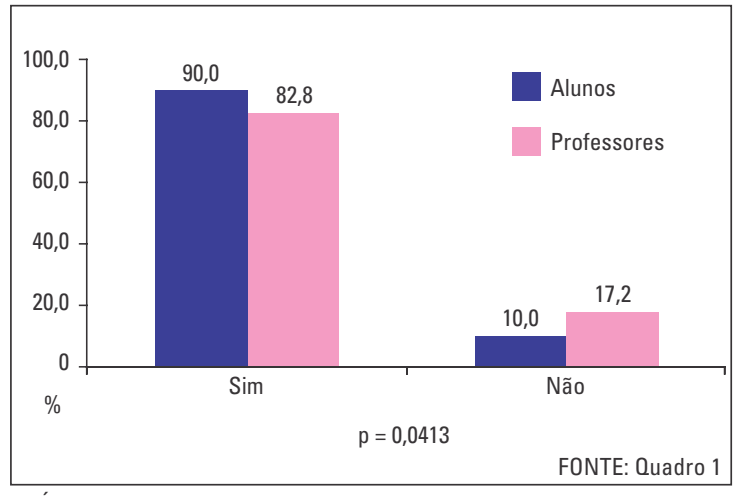

GRÁFICO 3 - Medidas proibitivas de cursos não regulamentados em relação aos alunos e professores.

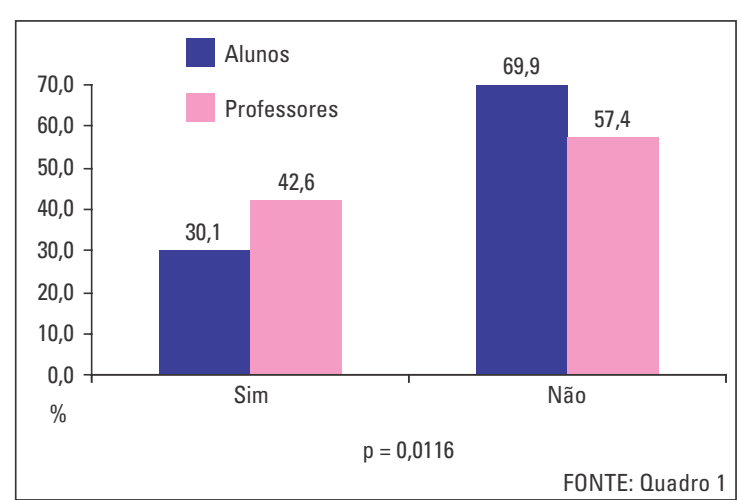

GRÁFICO 4 - Estimularia seu filho a estudar odontologia hoje em relação aos alunos e professores.

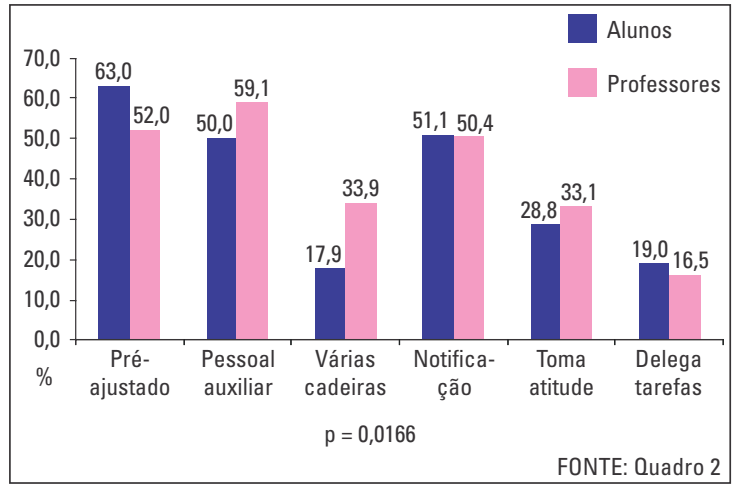

GRÁFICO 5 - Clínica mais eficiente quanto à duração do tratamento em relação aos alunos e professores.

\begin{tabular}{|c|c|c|c|c|c|c|}
\hline \multicolumn{7}{|c|}{ Quadro 3 - Estatística descritiva da idade e do tempo de formado e de exercício da especialidade (Ortodontia). } \\
\hline DADOS & N. ${ }^{\circ}$ & MÉDIA & $\begin{array}{l}\text { DESVIO PA- } \\
\text { DRÃOO }\end{array}$ & MíNIMO & MÁXIMO & MEDIANA \\
\hline Idade & 543 & 33,7 & 8,5 & 21,0 & 68,0 & - \\
\hline -Alunos & 413 & 30,8 & 5,3 & 21,0 & 50,0 & - \\
\hline -Professores & 130 & 42,8 & 10,1 & 25,0 & 68,0 & - \\
\hline Tempo de Formado & 540 & 11,0 & (1) 8,2 & 0,0 & 46,0 & 9,0 \\
\hline - Alunos & 410 & 8,1 & (1) 5,1 & 0,0 & 26,0 & 7,0 \\
\hline - Professores & 130 & 20,2 & (1) 9,2 & 4,0 & 46,0 & 18,0 \\
\hline Tempo de Ortodontia & 516 & 6,5 & (1) 6,9 & 0,0 & 35,0 & 4,0 \\
\hline - Alunos & 387 & 3,6 & (1) 3,1 & 0,0 & 17,0 & 3,0 \\
\hline - Professores & 129 & 15,0 & (1) 8,2 & 1,0 & 35,0 & 13,0 \\
\hline
\end{tabular}

(1) Desvio padrão muito elevado, recomenda-se utilizar a mediana. 
de Especialista (Quadro 3).

$\mathrm{Na}$ comparação dos alunos com os professores em estudo, foram constatadas as seguintes significâncias estatísticas para:

\section{Alunos}

- Acham cabível um controle de cursos não oficiais por Entidades e Órgãos competentes $(91,2 \%)(\mathrm{p}=0,0001)$ (Gráf. 2);

- Acham viáveis medidas proibitivas de cursos não regulamentados $(90,0 \%)(\mathrm{p}=0,0413)$ (Gráf. 3);

- Não estimularia seu filho a estudar Odontologia hoje $(69,9 \%)(\mathrm{p}=0,0116)$ (Gráf. 4);

- Tornam sua clínica mais eficiente em relação à duração do tratamento utilizando aparelhos préajustados $(63,0 \%)(p=0,0166)$ (Gráf. 5);

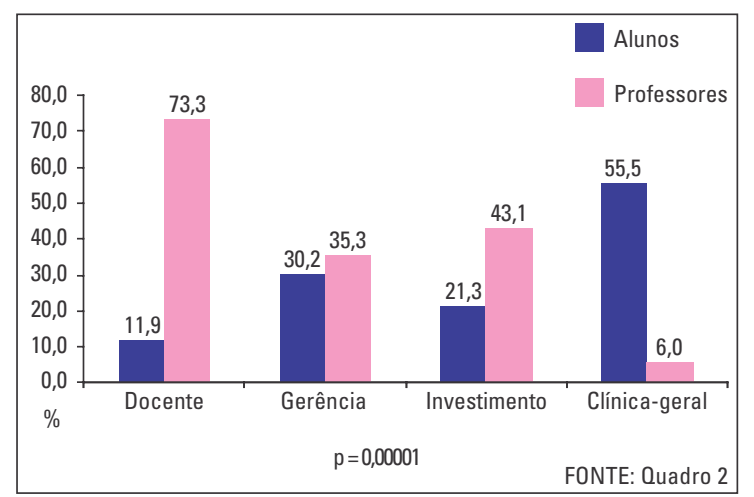

GRÁFICO 6 - Soluções e/ou alternativas para o incremento da renda em relação aos alunos e professores.

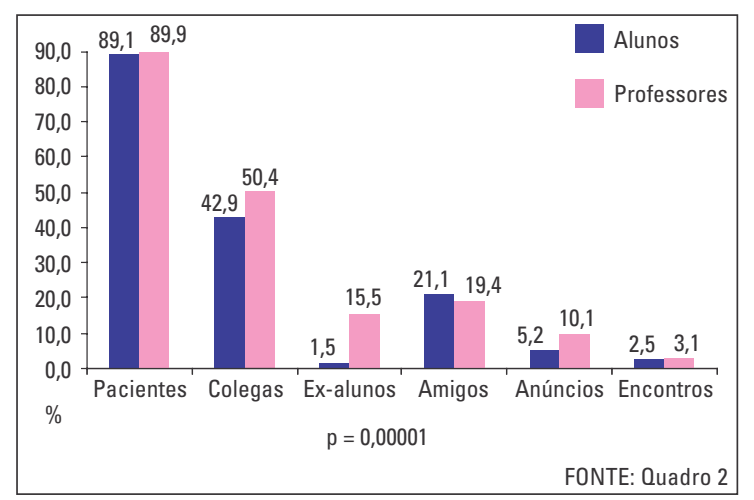

GRÁFICO 8 - Principal fonte de indicação de novos pacientes em relação aos alunos e professores.

\section{Professores}

- A solução para o incremento de sua renda é a atividade docente $(73,3 \%)(p<0,00001)$ (Gráf. 6);

- Acham correto estipular valores para o tratamento como um todo $(78,7 \%)(\mathrm{p}=0,0004)$ (Gráf. 7);

- A principal fonte de indicação de novos paciente são os próprios pacientes $(89,9 \%)$ $(\mathrm{p}<0,00001)$ (Gráf. 8);

-Utilizam como instrumentos de marketing os indicadores profissionais $(52,0 \%)(\mathrm{p}=0,0076)$ (Gráf. 9);

- São mais velhos $(p<0,0001)$, mais tempo de formado $(\mathrm{p}<0,0001)$ e conseqüentemente tem mais tempo de especialista em Ortodontia $(\mathrm{p}<0,0001)$.

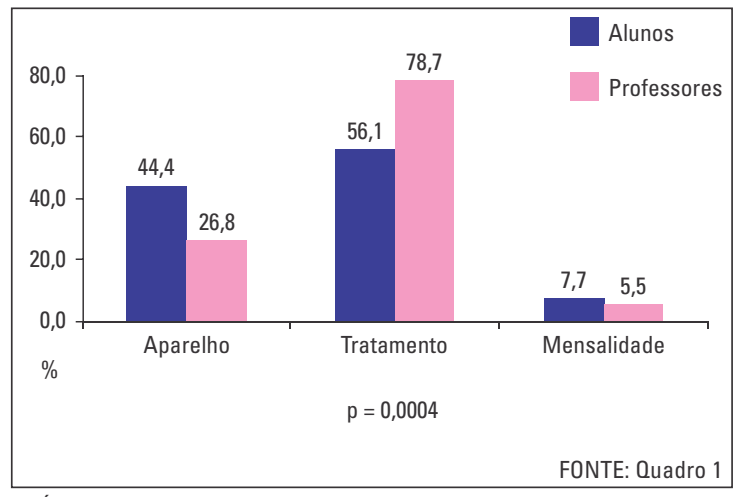

GRÁFICO 7 - 0 que acham correto em relação aos alunos e professores.

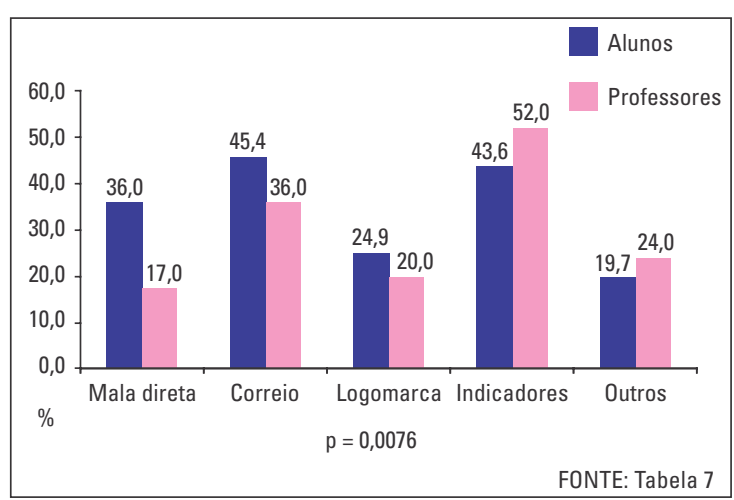

GRÁFICO 9 - Instrumento de marketing utilizado em relação aos alunos e Professores. 


\begin{tabular}{|c|c|c|}
\hline \multicolumn{3}{|c|}{ Tabela 9 - Idade média ortodontistas. } \\
\hline Estudo & $\begin{array}{l}\text { Idade } \\
\text { média }\end{array}$ & Autores \\
\hline I Estudo & 42 & QUEIROZ JR², 1993 \\
\hline II Estudo & 44,16 & PISSETTE ${ }^{30}, 1995$ \\
\hline Austrália & 43 & LAWRENCE, WRIGHT \& D'ADAMO 21,1995 \\
\hline III Estudo & 43,7 & SOUZA $^{33}, 1998$ \\
\hline $\mathrm{JCO}$ & 48 & GOTTLIEB, NELSON \& VOGELS ${ }^{13}, 1997$ \\
\hline Inglaterra & 42,6 & FOX et al. ${ }^{11}, 1997$ \\
\hline JCO & 49 & GOTTLIEB, NELSON \& VOGELS ${ }^{14,15}, 1999$ \\
\hline Caminhos* & 42,8 & MORGENSTERN²6, 2002 \\
\hline
\end{tabular}

* Relativo aos professores da pesquisa.

\begin{tabular}{|c|c|c|}
\hline \multicolumn{3}{|c|}{ Tabela 10 - Indicação. } \\
\hline Fonte & Pacientes & Colegas \\
\hline QUEIROZ JR², 1994 & 42,0 & 44,34 \\
\hline PISSETTE ${ }^{30}, 1997$ & 42,0 & 28,06 \\
\hline SOUZA $^{33}, 1998$ & 40,0 & -- \\
\hline GOTTLIEB' ${ }^{13}, 1997$ & 30,0 & 50,0 \\
\hline GOTTLIEB $^{14,15} 1999$ & 30,0 & 50,0 \\
\hline MORGENSTERN ${ }^{26}, 2002$ & 89,3 & 44,7 \\
\hline
\end{tabular}

\section{DISCUSSÃO}

\section{Idade}

A média de idade dos professores foi 42,8 , próxima das encontradas nos estudos com especialistas brasileiros e estrangeiros, demonstrados na tabela 9 .

\section{Como vê o mercado atual}

Para 58,7\% dos entrevistados, o mercado encontra-se saturado. Atualmente se constata o fechamento de alguns cursos de Odontologia.

\section{Causas da saturação do mercado}

As principais causas relacionadas pelos participantes foram o grande número de cursos não oficiais $(85,1 \%)$ e a concorrência por clínicos-gerais $(77,2 \%)$. Para Haag ${ }^{16}$ o que motivou os praticantes da especialidade foram o grande número de cursos irregulares $(81,17 \%)$ e a ilusão de lucro fácil e rápido $(77,64 \%)$. Na medida em que continuem presentes no Brasil, fatores como: 1) o enorme número de cursos de Ortodontia; 2) a intensa proliferação de cursos de técnica ortodôntica; 3 ) a abertura de novas faculdades; e 4) a crise financeira, nós teremos num prazo de 10-15 anos um decréscimo no número de pacientes atendidos e conseqüentemente da renda.

\section{Controle e medidas proibitivas de cursos não- regulamentados}

Conforme o quadro 1 , professores desejam menos o fechamento de cursos que alunos, o que denotou o maior interesse didático e financeiro destes. Uma das saídas para a Ortodontia é uma maior participação dos profissionais nas Entidades de Classe, nas Secretarias do Governo e no CFO, para atuar na gestão/ aprovação de Faculdades e Cursos de Especialização, combatendo cursos mercantilistas.

\section{Soluções/Alternativas para o incremento da renda}

Professores incrementam a renda com atividades docentes, e alunos com a clínica-geral, de acordo com a quadro 1 . Os convênios já possuem uma conotação social e econômica muito relevante na área da saúde no Brasil. Através das entidades representativas, em parceria com o Estado, devemos definir regras para a garantia da qualidade nos atendimentos e remuneração justa. Uma das saídas para absorver os profissionais no mercado de trabalho é a instituição de politicas governamentais mais adequadas, principalmente no Programa Saúde da Família (PSF) ou Centros de Referência para Especialidades.

\section{Como estipular valores}

De acordo com o quadro 1, professores cobram mais o tratamento como um todo $(78,7 \%)$ enquanto alunos dividem-se em cobrar o tratamento $(56,1 \%)$ e o aparelho $(44,4 \%)$.

\section{Honorários}

Foram considerados mais satisfatórios para os alunos da região $\mathrm{CO}+\mathrm{Ne}(58,2 \%)$ e para os professores de ambas regiões. 


\section{Estimularia seu filho a estudar Odontologia hoje?}

Alunos foram um pouco mais negativos do que professores, conforme o quadro 1.

\section{Tempo médio de tratamento com aparelha- gem fixa}

Alunos estimam menor tempo, enquanto professores são um pouco mais cautelosos, de acordo com a quadro 2 .

\section{Abordagem em relação ao paciente com den- tição mista}

Dos $96,5 \%$ respondentes, a maioria trata em 2 fases: $86,1 \%$.

\section{Instrumentos de Marketing utilizados}

Segundo Souza ${ }^{33}$, o marketing é considerado importante para $88,41 \%$ dos ortodontistas. Devem-se intensificar nos cursos de Especialização, a formação de profissionais com conhecimento de marketing e gerenciamento, visando o incremento da produtividade e qualidade na clínica diária.

\section{Fonte de indicação de novos pacientes}

Pacientes foram apontados como a maior fonte de referência $(89,3)$, valor bem superior às outras pesquisas, de acordo com a tabela 10 .

\section{Casuística no tratamento de adultos}

A casuística de $30 \%$ de adultos foi relatada por $29,7 \%$ dos profissionais.

Pissette $^{28}$ e Souza ${ }^{31}$ acharam o valor de 23,78\%; Gottlieb, Nelson, Vogels ${ }^{12}$, 24\% em 1987, 15,4\% em $1997^{13}$, e $15,5 \%$ em $1999^{14,15}$.

\section{Clínica mais eficiente em relação à duração do tratamento}

Alunos valem-se mais de aparelhos pré-ajustados $(63,3 \%)$, e professores de pessoal auxiliar. Notificação por escrito é utilizada por 50,9\%.

Falta de documentação dos casos durante fases intermediária e final
Cerca de $82,8 \%$ dos profissionais não estão documentando. Os alunos mais $(84,7)$ do que os professores $(76,7)$. As causas mais apontadas foram: 1) o custo para o paciente $(61,9)$ e 2$)$ a falta de interesse do profissional $(21,0)$.

\section{CONCLUSÕES}

- A mediana de idade do aluno foi 30,8; e a do professor 42,8 .

- São as causas principais da saturação do mercado: o grande número de cursos não oficiais $(85,1)$ e a concorrência por clínicos-gerais $(63,4)$.

- A maioria deseja o controle de cursos não oficiais $(87,9)$ e medidas proibitivas de cursos não regulamentados $(88,3)$.

- Professores incrementam sua renda com atividades docentes $(73,3)$ e alunos com clínica-geral $(55,5)$.

- Cobrar o tratamento como um todo é adotado por $61,6 \%$.

- Honorários são insuficientes para 44,8\%, satisfatórios para 44,3\% e bons para 5,9\%.

- Orientar o filho a estudar Odontologia é menos estimulado por alunos $(69,9)$ do que por professores $(57,4)$.

- O tempo de tratamento de 30 meses $(47,6)$ foi o mais estimado, seguido de 24 meses $(41,6 \%)$.

- A grande maioria trata a Dentição Mista em 2 fases $(86,1)$.

- A maior fonte de indicação são os próprios pacientes $(89,9)$.

- Cerca de 29,7\% dos profissionais tratam 30\% de adultos.

- Alunos usam mais aparelhos pré-ajustados $(63,0)$ e professores trabalham mais com auxiliares $(59,1)$.

- Professores utilizam mais indicadores profissionais $(52,0)$; e alunos, correspondências festivas $(45,4)$.

- A propaganda "boca a boca" foi largamente relacionada como instrumento de marketing $(47,6)$.

- $82,8 \%$ não estão documentando fases intermediária e final. 


\title{
Orthodontics - march and countermarch in Brazil
}

\begin{abstract}
Due to the increase in the number of orthodontists and Graduate Programs, this study was outlined aiming to the future of our Speciality. Questionaires were sent to 413 students and 130 teachers in 42 Graduate Programs. Respondents achieved 57.53\%. The sample included respondents from several areas in Brazil. The average age of the students was 30.8 and the teachers' 42,8 . The average index suggested by the Health World Organization is 1 dentist for each 1.500 habitants. Brazil has a population of 175 millions people and has 175 thousand dentists, one for each 996 inhabitants. This proportion in England is 1/2.000, in France 1/1.519, in Italy $1 / 1.333$. With a populacion $40 \%$ smaller and income $82 \%$ smaller than the USA, Brazil holds almost three times the amount of Orthodontic Courses (124, 2001), graduating 344 ortodontists/ year. Considering the 175.637 dentists, the specialists number still is little in Brazil. Among orthodontists, $58.5 \%$ consider the professional market satureted due to non official courses $(85,1 \%)$ and to the competition with general practioners $(63,4 \%)$. Besides, $88,3 \%$ of the orthodontists would favor rules to inhibit irregular courses. The increase of their incomes comes from the general practice by the students $(58,2 \%)$ while $73,3 \%$ of the teachers raise their income through teaching activities. The fees are considered insuficient by $44.85 \%$ of the orthodontists and satisfactory by $47.3 \%$ of them. Nowadays, $66.9 \%$ of these specialists would not encourage their children to study Dentristy. The main referral source comes from patients (89.3\%). Another rmarkable issue is that intermediate and final records have not being taken by $82.8 \%$ of the orthodontists.
\end{abstract}

Key words: Brazilian orthodontists. Professional market. Office management.

\section{REFERÊNCIAS}

1. ASSOCIAÇÃO BRASILEIRA DE ODONTOLOGIA - ABO. Responsabilidade de grupo. Revista ABO Nacional, São Paulo, v. 8, n. 6, p. 332, dez. $2000 /$ jan. 2001.

2. ASSOCIAÇÃO BRASILEIRA DE ORTODONTIA E ORTOPEDIA FACIAL. Catálogo ABOR: 1999/2000, Maringá: Dental Press Internacional, 1999, p. 263-270.

3. BERGSTRÖM, Kurt et al. Treatment difficulty and treatment outcome in orthodontic care. Eur Orthod Society, v. 20, p. 145-157, 1998

4. CAUDURO, Ricardo. De quem é a culpa? Editorial. Revista Gaúcha de Odontologia, Porto Alegre, v. 48 , n. 4, p. 1, out./ dez. 2000.

5. CONSELHO FEDERAL DE ODONTOLOGIA. 2a Aneo: debate sobre especialidades começou. Jornal do Conselho Federal de Odontologia. Rio de Janeiro, ano 9, n. 43, p. 6-7, mar./ abr. 2001.

6. CONSELHO FEDERAL DE ODONTOLOGIA. Números do CFO em todos os Estados. Disponível em: <http://www.cfo.org.br> Acesso em: 14 set. 2001

7. CONSELHO REGIONAL DE ODONTOLOGIA DO PARANÁ. Cirurgiões- dentistas se mobilizam contra política educacional do governo federal. Revista CRO-PR, Curitiba, ano 5, n. 20, p. 13, maio 1999

8. COORDENAÇÃO DE APERFEIÇOAMENTO DE PESSOAL DE NÍVEL SUPERIOR. Números de Mestrados e Doutorados em Ortodontia. Disponível em: <http://www.capes.org.br> Acesso em: 14 set. 2001

9. FALTIN JÚNIOR, Kurt. Manifesto da ABOR à Ortodontia e Ortopedia Facial Brasileira à Odontologia e ao público em geral. Jornal da APCD, São Paulo, ano 36, n. 531, p. 47, jul. 2001.
10. FERES, Marco A. L. Ortodontia: torne seu consultório, sua vida e você mais simples e mais eficientes. In: FERES, Marco A. L. (Coord.). Ortodontia: algumas histórias de sucesso. 1. ed. Curitiba: Editek, 1999. p. 133-138.

11. FOX, N. A. Factors affecting the outcome of orthodontic treatment within the general dental service. Br J Orthod, London, v. 24 , no. 3 , p. 217-221, Ago. 1997.

12. GOTTLIEB, Eugene L.; NELSON, Allen H.; VOGELS, David S. JCO orthodontic practice study - part 1. J Clin Orthod, Boulder, v. 21 , no. 8 , p. 507-515, Aug. 1987.

13. $1997 \mathrm{JCO}$ orthodontic practice study part 2 practice success. J Clin Orthod, Boulder, v. 31, no. 11, p. 741-748, Nov. 1997.

14. _ 1999 JCO orthodontic practice study. Part 2 - practice success. J Clin Orthod, Boulder, v. 33, no. 11, p. 627-635, Nov. 1999.

15. 1999 JCO Orthodontic Practice Study part 3 Practice Growth. J Clin Orthod, Boulder, v. 33, no. 12, p. 675-688, Dec. 1999.

16. HAAG, Celso Antonio. Aspectos éticos e legais da Ortodontia no Brasil. Ortodontia, São Paulo, v. 32, n. 2, p. 67-81, maio/ ago. 1999.

17. ISIDORO, Fernanda. Dilalogo aberto com o MEC. Jornal do Conselho Federal de Odontologia, Rio de Janeiro, ano 9, n. 44, p. 6-7, maio/jun. 2001.

18. JONHSON, David A.; GOTTLIEB, Eugene L.; DOMER, Larry R. JCO Orthodontic practice study - practice success. J Clin Orthod, Boulder, v. 15, no. 10, p. 683-693, Oct. 1981.

19. KELLY, B. M.; SPRINGATE, S. D. Specialist in the General Dental Service. Br Dental J, London, v. 180, no. 6, p. $209-215$, Mar. 1996 
20. KOUBIK, Roberto; FERES, Marco A. L. Aspectos legais da Ortodontia. Ortodontia, São Paulo, v. 28, n. 2, p. 64-70, jun./ ago. 1995.

21. LAWRENCE, A. J. ; WRIGHT, F. A. C.; D'ADAMO, S. P. The provision of orthodontics services by general dental practioners. 2. Factors influencing variation in service provision. Aust Dental J St. Leonards, v. 40, no. 6, p. 360-364, June 1995.

22. LIMA, Israel Correia de. Odontologia: um mercado cada vez mais difícil. Jornal da Associação Paulista de Cirurgiões-Dentistas, São Paulo, ano 35, n. 520, p. 26-27, ago. 2000

23. __. Atual conjuntura econômica é favorável ao profissional liberal. Jornal da Associação Paulista de Cirurgiões-Dentistas São Paulo, ano 35, n. 527, p. 20-21, mar. 2001.

24. Sobram escolas de Odontologia e faltam alunos. Jornal da Associação Paulista de Cirurgiões-Dentistas, São Paulo, ano 36, n. 530, p. 32, jun. 2001.

25. A semelhança entre a abertura de cursos de Odontologia e Medicina. Jornal da Associação Paulista de Cirurgiões-Dentistas, São Paulo, ano 36, n. 534, p. 36, 2001.

26 MORGENSTERN, Anna Paula Morgenstern. Caminhos e descaminhos da Ortodontia. 2002. 143 f. Monografia (Especialização em Ortodontia) - Setor de Ciências da Saúde, Universidade Federal do Paraná, Curitiba, 2002.

27. PETRELLI, Eros. O engodo dos certificados. Jornal da Sociedade Paulista de Ortodontia, São Paulo, ano 5, n. 24, p. 7, mar./ abr. 1993.

28. Os eunucos da Odontologia. Jornal da Sociedade Paulista de Ortodontia, São Paulo, ano 5, n. 30, p.10, mar./ abr. 1994.
29. PETRELLI, Eros. Assembléia Nacional das Especialidades Odontológicas. Conselho Regional de Odontologia do Paraná, Curitiba, ano 7, n. 32, p. 17, maio/ jun. 2001.

30. PISSETTE, Ana Paula. II Estudo sobre a prática ortodôntica no Brasil - 1995. Ortodontia, São Paulo, v. 30, n. 3, p. 7-15, set./ dez. 1997.

31. QUEIROZ JUNIOR, Geraldo. Estudo sobre a prática ortodôntica no Brasil. Ortodontia, São Paulo, v. 27, n. 3, p. 67-77, set./ dez. 1994.

32. RICHMOND, S.; FOX, N.; WRIGHT, J. The professional perception of orthodontic treatment complexity. Br Dent J, London, v. 183 , no. 10 , p. 365-370, Nov. 1997.

33. SOUZA, Carlos Eduardo Vieira. III Estudo sobre a prática ortodôntica no Brasil - 1998. 1998. 101 f. Monografia (Especialização em Ortodontia) - Setor de Ciências da Saúde, Universidade Federal do Paraná, Curitiba, 1998.

34. STRATFORD, N. M.; BURDEN, D. J. Clinical assistant training in orthodontics: how effective is it? Br Dental J, London, v. 184, no. 9, p. 448-452, May 1998.

35. SULLIVAN, Edward F. Overhead Reduction: is bigger always better? J Clin Orthod, Boulder, v. 28, no. 1, p. 21-30, Jan. 1994.

36. WALDMAN, H. Barry. Changing number and distribution of orthodontists: 1987-1995. Am J Orthod Dentofacial Orthop St. Louis, v. 114, no. 1, p. 50-54, July 1998.

37. WIDSTRÖM, K. A. et al. Oral heathcare in transition in Eastern Europe. Br Dent J, London, v. 190, no. 11, p. 580-584, June 2001.
Endereço para correspondência

Anna Paula Morgenstern

Rua Martin Afonso, n. 1197, ap.31

Champagnat - Curitiba - Paraná

CEP: 80430-100 